\title{
A large and pedunculated inflammatory pseudotumor with pseudosarcomatous change of the cecum mimicking a malignant polyp: a case report and literature review
}

\author{
Jong Suk Oh, Hyung Wook Kim, Su Bum Park, Dae Hwan Kang, Cheol Woong Choi, Su Jin Kim, Hyeong Seok Nam, \\ Dae Gon Ryu
}

Department of Internal Medicine, Pusan National University School of Medicine and Research Institute for Convergence of Biomedical Science and Technology, Pusan National University Yangsan Hospital, Yangsan, Korea

Inflammatory pseudotumor (IPT) is a rare benign tumor of unknown etiology that can occur in almost any organ system. It has neoplastic features such as local recurrence, invasive growth, and vascular invasion, leading to the possibility of malignant sarcomatous changes. The clinical presentations of colonic IPT may include abdominal pain, anemia, a palpable mass, and intestinal obstruction. A few cases of colonic IPT have been reported, but colonic IPT with pedunculated morphology is very rare. Furthermore, since it can mimic malignant polyps, understanding the endoscopic findings of colonic IPT is important for proper treatment. Herein, we present a case of colonic IPT with pseudosarcomatous changes, presenting as a large polyp, mimicking a malignant polyp in the cecum, along with a literature review.

Keywords: Cecum; Inflammatory pseudotumor; Pedunculated polyp

\section{INTRODUCTION}

Inflammatory pseudotumor (IPT) is a rare benign tumor that has been synonymously referred to as a plasma cell granuloma or inflammatory myofibroblastic tumor. ${ }^{1}$ IPTs occur most commonly in the lung and the orbit of the eye. However, they have been reported to occur in almost every organ system. ${ }^{2}$ The exact etiology remains obscure. IPT has been associated with infections, such as the Epstein-Barr virus and human herpes vi-

Received: February 24, 2021 Revised: March 31, 2021

Accepted: April 5, 2021

Correspondence: Hyung Wook Kim

Department of Internal Medicine, Pusan National University Yangsan

Hospital, Pusan National University School of Medicine and Research Institute for Convergence of Biomedical Science and Technology, 20 Geumoro, Mulgeum-eup, Yangsan 50612, Korea

E-mail: mdkhwook@gmail.com

(a) This is an Open Access article distributed under the terms of the Creative Commons Attribution Non-Commercial License (http://creativecommons.org/licenses/by-nc/4.0/) which permits unrestricted non-commercial use, distribution, and reproduction in any medium, provided the original work is properly cited. rus, and is related to reactive cytokine production and previous abdominal surgery or trauma. ${ }^{3,4}$ Clinical presentation depends upon the affected organ. A palpable mass, fever, weight loss, malaise, pain, and site-specific symptoms are the most common presenting complaints or manifestations. ${ }^{5}$ Colonic IPT is a rare condition and can be mistaken for colon cancer. ${ }^{6}$ Among colonic IPTs, colonic IPT with a pedunculated morphology is even rarer.

Here, we report an uncommon case of colonic IPT presenting as a large pedunculated polyp, mimicking a malignant polyp in the cecum. We also reviewed the endoscopic findings of reported cases in the literature.

\section{CASE REPORT}

A 32-year-old female patient with no underlying disease was admitted to a local clinic complaining of right lower quadrant pain that persisted for 2 weeks. Abdominal computed tomography (CT) performed at a local hospital revealed a soft-tissue 
mass, measuring approximately $4 \mathrm{~cm}$, and increased colonic wall thickness in the ascending colon and cecum (Fig. 1). As her symptoms did not improve following antibiotics for infectious colitis, she was referred to the outpatient gastroenterology clinic in our hospital for further evaluation. She had an acutely ill-looking appearance. Physical examination revealed tenderness in the right lower quadrant. Laboratory analysis revealed that hemoglobin, total leukocyte count, platelet count, and segmental neutrophils were $13 \mathrm{~g} / \mathrm{dL}$ (reference range, 11.5-15.5 g/ $\mathrm{dL}$ ), $9.28 \times 10^{3} / \mathrm{dL}$ (reference range, $4.0-11.0 \times 10^{3} / \mathrm{dL}$ ), $502 \times 10^{3} /$
$\mathrm{dL}$ (reference range, $140-400 \times 10^{3} / \mathrm{dL}$ ), and $67.9 \%$ (reference range, $40 \%-75 \%$ ), respectively. Other chemical tests and tumor markers were within normal limits, except for a slight elevation of C-reactive protein $(0.85 \mathrm{mg} / \mathrm{dL})$. The patient underwent colonoscopy for evaluation of the colonic mass. Colonoscopy revealed an approximately $4-5 \mathrm{~cm}$ polypoid mass with a short stalk, thickly coated exudates, mucosal desquamation, and deep ulcers in the ascending colon and cecum (Fig. 2). A malignant polyp originating from an adenocarcinoma was suspected initially. Other malignant tumors, such as lymphoma and gastro-
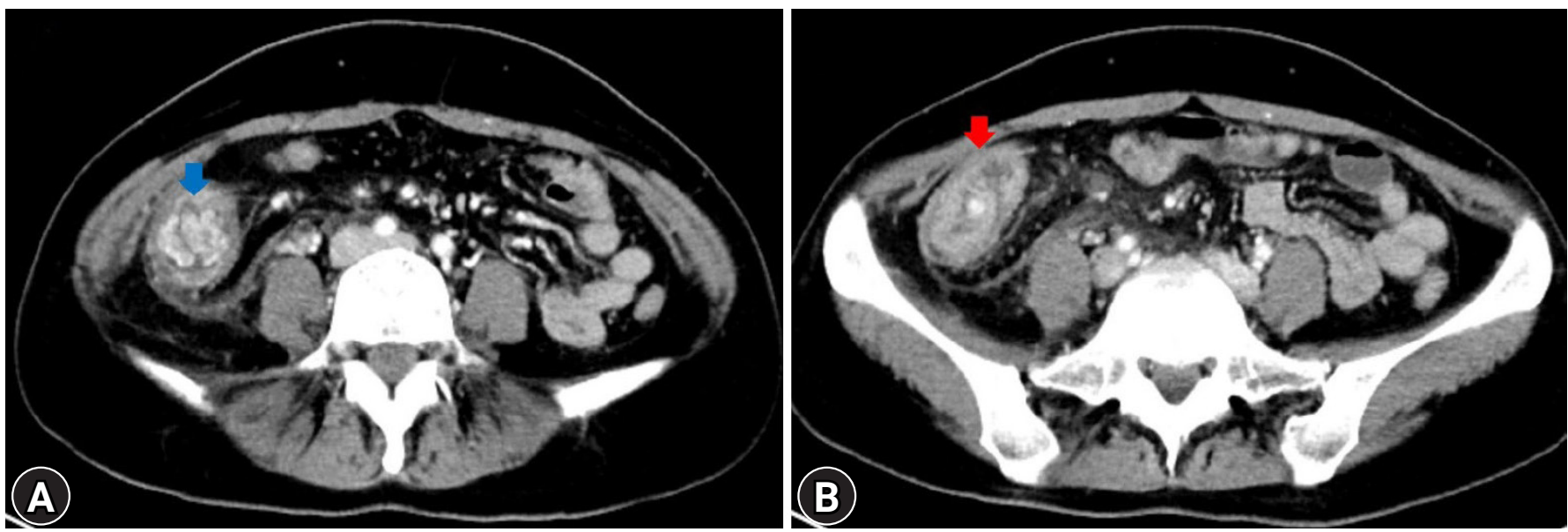

Fig. 1. Abdominal computed tomography images. (A) A well-enhancing soft-tissue mass (blue arrow), measuring approximately $4 \mathrm{~cm}$, at the ascending colon. (B) Increased colonic wall thickness (red arrow) of the ascending colon and cecum.
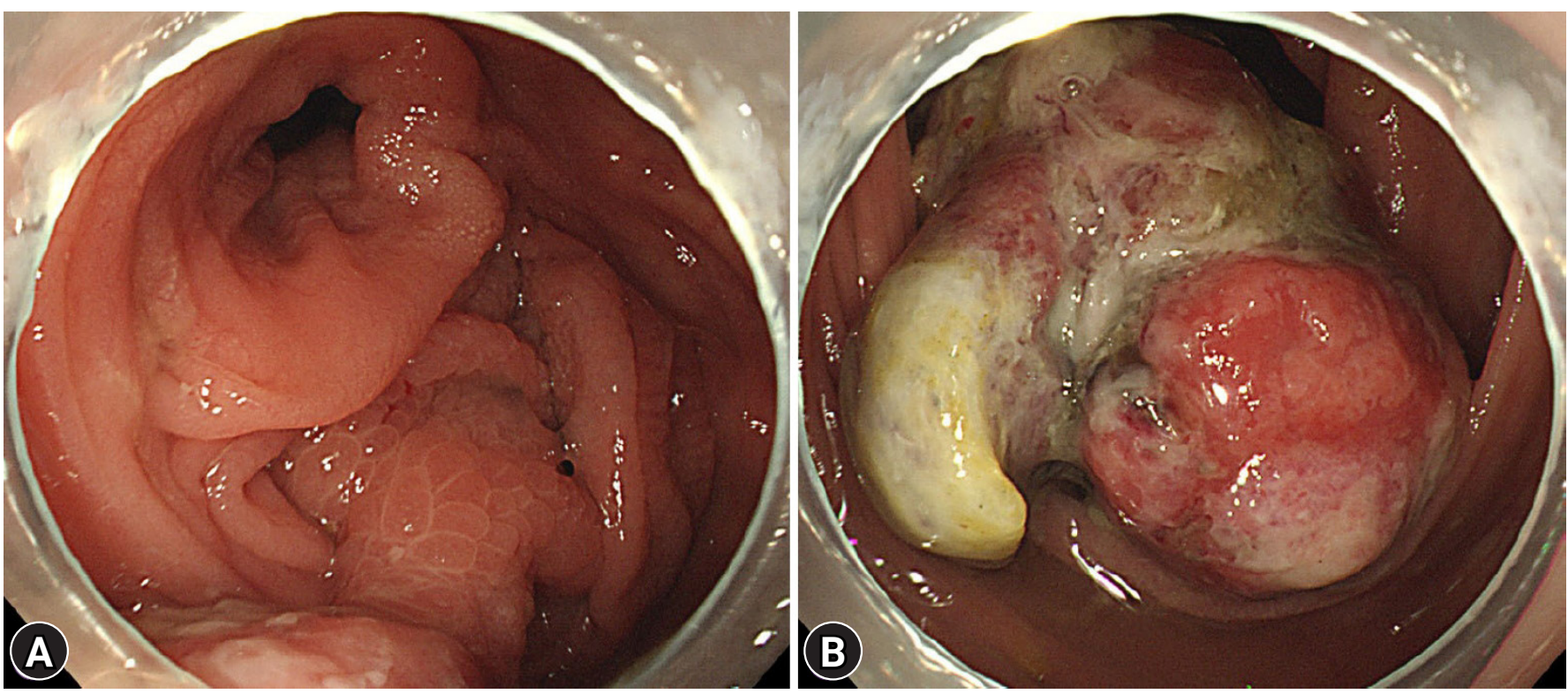

Fig. 2. Colonoscopic findings. (A, B) An approximately 4-5 cm polypoid mass with a short stalk, thickly coated exudates, mucosal desquamation, and deep ulcers originating in the cecum. 
intestinal stromal tumor, were included as differential diagnoses because of the atypical endoscopic findings. Multiple biopsies of the desquamated surface and ulcers of the mass were obtained. The biopsy results showed marked pleomorphic and spindle cell proliferation with severe neutrophil infiltration. A pseudosarcomatous stroma of the IPT was suspected. A laparoscopic right hemicolectomy was performed because the abdominal pain became more frequent and more severe while the patient was waiting for an outpatient review. The biopsy alone could not completely exclude malignancy. Grossly, the resected specimen revealed a $4.5 \times 3.8 \times 2.2 \mathrm{~cm}$ polypoid mass with a short stalk originating in the cecum (Fig. 3A). Microscopic examination revealed marked pleomorphic cell proliferation limited only to the mucosa and submucosa, with ulceration evident at low magnification (Fig. 3B) and marked pleomorphic and spindle cell proliferation with severe neutrophil infiltration at higher magnification (Fig. 3C). Based on the histological features, the mass was diagnosed as an IPT with pseudosarcomatous changes. There was no infiltration of the local lymph nodes.

The patient's postoperative course was uneventful, and she was discharged on the fifth postoperative day. She did not have any complications during the 8-month follow-up period.

\section{DISCUSSION}

IPT is a rare benign lesion. Histologically, it is characterized by dominant spindle cell proliferation with acute and chronic inflammatory cells. ${ }^{7}$ IPT may occur at any age with no sex predilection. ${ }^{8}$ Although IPT is a benign lesion, it presents with neoplastic features such as local recurrence, infiltrative growth, and vascular invasion, and therefore, there is a possibility of malignant sarcomatous changes.
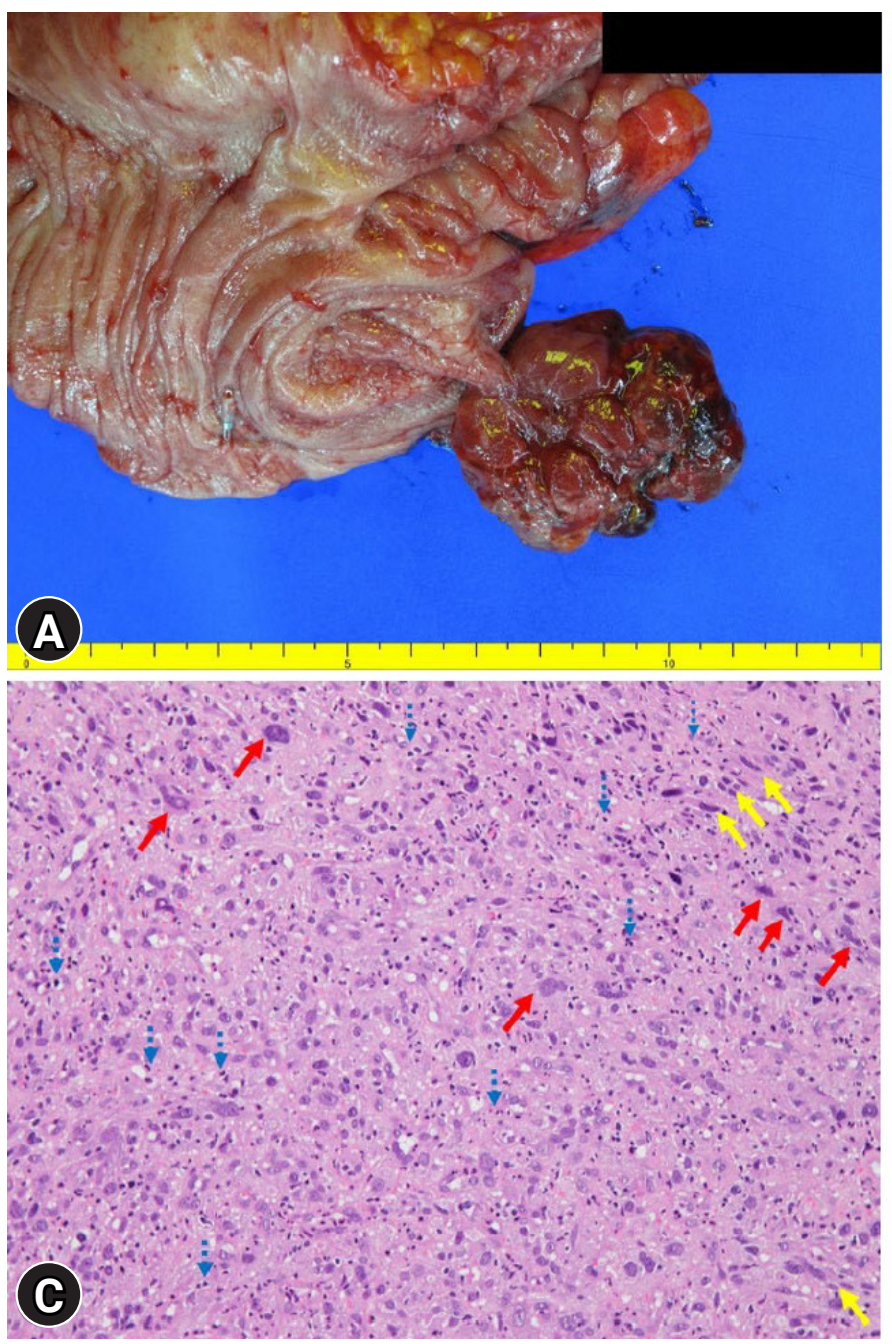

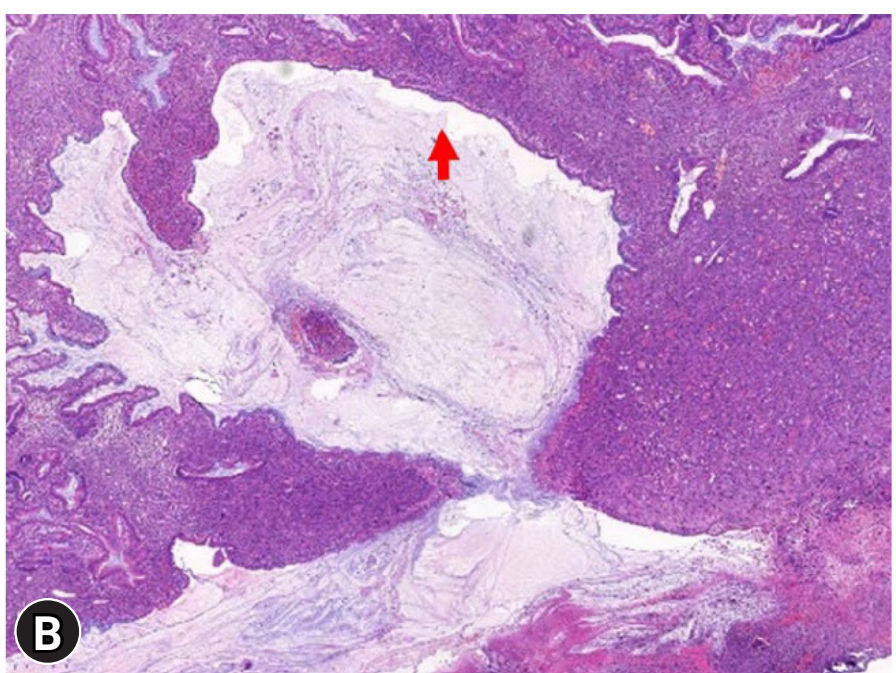

Fig. 3. Gross and pathologic findings. (A) A well-defined pedunculated mass at the cecum. (B) Ulceration (red arrow) with marked pleomorphic cell proliferation limited to the mucosa and submucosa (hematoxylin and eosin stain, $\times 6$ ). (C) Marked pleomorphic cell (red arrows) and spindle cell proliferation (yellow arrows) with severe neutrophil infiltration (blue dotted arrows) (hematoxylin and eosin stain, $\times 200)$. 
Colonic IPT is rare. In the study by Höhne et al., ${ }^{6}$ only 17 cases $(1.7 \%)$ of colonic IPTs were identified among a total of 956 IPT cases. Although its clinical symptoms are diverse depending on the location of the lesion, abdominal pain, a palpable mass, and occasionally intestinal obstruction are the most common signs and symptoms. ${ }^{2,5}$ Recently, Karaisli et al. ${ }^{10}$ assessed 60 patients with colonic IPTs and presented a comprehensive review of the literature. In the literature review, a male predominance (24 males and 15 females) was reported. Age-specific analysis revealed the highest incidence rates in patients aged 0-18 years and 30-50 years. Preoperative or intraoperative biopsy was performed in only $13(21.7 \%)$ patients. The ascending colon $(25 \%)$ was the most common location. The mean tumor diameter was found to be $6.21 \pm 3.31 \mathrm{~cm}$. Regarding management, 59 (98.3\%) out of 60 patients underwent surgical resections, and one patient with unresectable IPT was managed with high doses of nonsteroidal anti-inflammatory drugs (NSAIDs). Seven (14.3\%) out of 49 patients for whom follow-up data were available, the disease recurred locally. However, no distant metastasis was observed.

In our case, a 32-year-old female patient presented with intermittent abdominal pain in the right lower quadrant with intussusception. The preoperative colonoscopy and abdominal CT findings suggested a malignant polyp. The cause of IPT was obscured in our case. However, considering the abundant neutrophil and pleomorphic cell infiltration, an acute inflammation caused by bacterial or viral infection was suspected. The patient underwent surgical resection. The mass resected from the cecum was a polypoid lesion $(4.5 \times 3.8 \times 2.2 \mathrm{~cm})$ with a pedunculated morphology. Colonic IPTs are often misdiagnosed as colonic carcinoma on abdominal CT, especially in older adults. ${ }^{10}$ A correct preoperative diagnosis of this disease is difficult because the radiologic findings are variable and nonspecific. ${ }^{11}$ Therefore, preoperative colonoscopic biopsy is highly recommended for an accurate diagnosis. However, this may be difficult in many patients because of severe abdominal pain and bowel obstruction. Another reason for the need for preoperative colonoscopy is that endoscopic resection is possible in cases of small colonic IPT of pedunculated morphology. However, the data on endoscopic resection and endoscopic findings of colonic IPTs remain unclear.

Endoscopic differential diagnosis of colonic IPT includes colon cancer originating from adenocarcinomas and submucosal tumors, such as lymphoma and gastrointestinal stromal tumors. Our patient had two distinct endoscopic findings that are dif- ferent from those in previous reports. First, the surface of the mass was covered with normal mucosa, except for the ulcerated lesion. This finding differs from that of adenomas or adenocarcinomas. Second, colonic IPT with a pedunculated polyp is rare. In the literature review, only two cases had a pedunculated morphology. In our patient, the mucosal desquamation of the mass suggested a submucosal tumor, but the stalk of the mass suggested a malignant polyp originating from an adenocarcinoma. Table 1 shows the endoscopic findings of 11 cases of colon IPTs reported in the PubMed database between 2000 and 2020. ${ }^{8,10-17}$ Most of the lesions were large $(2-3 \mathrm{~cm})$ and polypoid in morphology. Two cases (No. 4 and 5) had a polypoid mass with a stalk, and two cases (No. 2 and 8) were accompanied by luminal stenosis due to infiltrative changes. Although there are differences in endoscopic findings depending on the time of diagnosis, most colonic IPTs have the characteristics of submucosal tumors and are accompanied by mucosal desquamation, ulceration, and depression over time. Therefore, colonic IPT should be considered when a patient presents with a large polypoid mass that resembles a submucosal tumor with surface changes, such as mucosal desquamation, ulceration, and depression. However, these endoscopic findings have also been observed in other malignant tumors. Hence, a colonoscopic biopsy is necessary for an accurate definitive diagnosis.

Surgical resection is the most important treatment option. NSAIDs, chemotherapy, radiation treatment, steroids, and cyclosporin A have been used as treatment modalities, but the benefits are still unclear. ${ }^{3,7}$ Treatment modalities other than surgery may be attempted if it is impossible to resect the IPT and if the patient has comorbidities that contraindicate surgery. It has been reported that regression of non-resectable intra-abdominal IPTs with NSAIDs may be possible. ${ }^{18}$ Response to steroids is often unpredictable. ${ }^{2}$ Colonic IPT may recur after surgical resection. According to Karaisli et al., ${ }^{10}$ colon IPT recurrence was confirmed in $14.3 \%$ of patients who were followed up after surgery. Local recurrence was detected from 2 months to 74 months after surgery, but $83.3 \%$ of recurrences were confirmed within 18 months after surgery. Therefore, close and regular follow-up according to surveillance guidelines for postoperative colorectal cancer is required for these patients.

We reported an uncommon case of colonic IPT with pseudosarcomatous changes presenting as a large pedunculated polyp, mimicking a malignant polyp in the cecum, and reviewed the endoscopic findings of the cases reported in the literature. Correlations between endoscopic findings and colonoscopic 
Table 1. Characteristics summary and endoscopic findings of colon IPT patients reported in the PubMed database from 2000 to 2020

\begin{tabular}{|c|c|c|c|c|c|c|}
\hline $\begin{array}{l}\text { Case } \\
\text { no. }\end{array}$ & Study & $\begin{array}{c}\text { Sex/age } \\
(\mathrm{yr})\end{array}$ & Location & $\begin{array}{l}\text { Diameter } \\
\text { (cm) }\end{array}$ & Endoscopic finding & Treatment \\
\hline 1 & Rosenbaum et al. $(2000)^{12}$ & $\mathrm{M} / 73$ & Sigmoid & 3.5 & Polypoid mass with nodular surfaces & Low anterior resection \\
\hline 2 & Nakamura et al. $(2010)^{13}$ & $\mathrm{~F} / 82$ & NA & NA & $\begin{array}{l}\text { Multiple, small polyps with luminal } \\
\text { stenosis }\end{array}$ & Observation and follow-up \\
\hline 3 & Tanaka et al. $(2010)^{14}$ & $\mathrm{M} / 79$ & Ascending & 2 & Sessile mass with central depression & Rt. hemicolectomy \\
\hline 4 & Tanaka et al. $(2010)^{14}$ & $\mathrm{M} / 79$ & Ascending & 2 & Polypoid mass with a short stalk & Rt. hemicolectomy \\
\hline 5 & Jeong et al. $(2011)^{11}$ & $\mathrm{~F} / 30$ & Splenic flexure & 5 & Polypoid mass with ulcers and a stalk & Lt. hemicolectomy \\
\hline 6 & Salameh et al. $(2011)^{15}$ & $\mathrm{M} / 2.6$ & Sigmoid & NA & Polypoid mass covered with exudates & Lt. hemicolectomy \\
\hline 7 & Kim et al. $(2012)^{8}$ & $\mathrm{M} / 35$ & Descending & 3.9 & Polypoid mass with shallow ulcers & Low anterior resection \\
\hline 8 & Satahoo et al. $(2013)^{16}$ & $\mathrm{M} / 14$ & Rectum & 6 & $\begin{array}{l}\text { Ulceroinfiltrative mass with luminal } \\
\text { stenosis }\end{array}$ & Low anterior resection \\
\hline 9 & Walia et al. $(2014)^{17}$ & $\mathrm{~F} / 10$ & Ascending & 5 & Polypoid mass with shallow ulcers & $\begin{array}{l}\text { Mid ascending colon segmental } \\
\text { resection }\end{array}$ \\
\hline 10 & Karaisli et al. $(2020)^{10}$ & $\mathrm{M} / 42$ & Cecum & 9 & Polypoid mass with nodular surfaces & Rt. hemicolectomy \\
\hline 11 & Karaisli et al. $(2020)^{10}$ & $\mathrm{M} / 40$ & Descending & 6.5 & Polypoid mass & Lt. hemicolectomy \\
\hline
\end{tabular}

IPT, inflammatory pseudotumor; M, male; F, female; NA, not available; Rt., right; Lt., left.

biopsy are important for proper treatment because it is difficult to radiologically differentiate colonic IPT from other malignant tumors, and endoscopic resection is possible in cases of small tumors or those with pedunculated morphology.

\section{Conflicts of Interest}

The authors have no potential conflicts of interest.

\section{Funding}

None.

\section{Author Contributions}

Conceptualization: HWK; Data curation: JSO, SBP, DHK, CWC, SJK, HSN, DGR; Writing-original draft: JSO; Writing-review \& editing: all authors.

\section{ORCID}

Jong Suk Oh Hyung Wook Kim Su Bum Park Dae Hwan Kang Cheol Woong Choi Su Jin Kim Hyeong Seok Nam Dae Gon Ryu

\section{REFERENCES}

1. Dehner LP. The enigmatic inflammatory pseudotumours: the current state of our understanding, or misunderstanding. J Pathol 2000;192:277-279.

2. Narla LD, Newman B, Spottswood SS, et al. Inflammatory pseudotumor. Radiographics 2003;23:719-729.

3. Karnak I, Senocak ME, Ciftci AO, et al. Inflammatory myofibroblastic tumor in children: diagnosis and treatment. J Pediatr Surg 2001;36:908-912.

4. Fletcher C, Senocak J, Fletcher C. Inflammatory myofibroblastic tumour: World Health Organization classification of tumours. IARC Press; Lyon, France: 2002.

5. Coffin CM, Watterson J, Priest JR, et al. Extrapulmonary inflammatory myofibroblastic tumor (inflammatory pseudotumor). A clinicopathologic and immunohistochemical study of 84 cases. Am J Surg Pathol 1995;19:859-872.

6. Höhne S, Milzsch M, Adams J, et al. Inflammatory pseudotumor (IPT) and inflammatory myofibroblastic tumor (IMT): a representative literature review occasioned by a rare IMT of the transverse colon in a 9-year-old child. Tumori 2015;101:249-256.

7. Kovach SJ, Fischer AC, Katzman PJ, et al. Inflammatory myofibroblastic tumors. J Surg Oncol 2006;94:385-391.

8. Kim EY, Lee IK, Lee YS, et al. Inflammatory myofibroblastic tumor in colon. J Korean Surg Soc 2012;82:45-49.

9. Patnana M, Sevrukov AB, Elsayes KM, et al. Inflammatory pseudotumor: the great mimicker. AJR Am J Roentgenol 2012;198:W217- 


\section{Geamenamer}

W227.

10. Karaisli S, Kamer E, Ekinci N, et al. Inflammatory myofibroblastic tumour of the colon: 2 case reports and a comprehensive review of the literature. Int J Colorectal Dis 2020;35:947-958.

11. Jeong JH, Cho IH, Kong EJ, et al. (18)F-FDG PET/CT in inflammatory pseudotumor of the colon causing intussusception. Ann Nucl Med 2011;25:447-450.

12. Rosenbaum A, Arnold JC, Rebel M, et al. Pseudotumor of the sigmoid mimicking carcinoma. Endoscopy 2000;32:546-548.

13. Nakamura Y, Kayano H, Shimada T, et al. Plasma cell granuloma of the sigmoid colon associated with diverticular disease and accompanying IgM-type monoclonal gammopathy. Intern Med 2010;49:227230.

14. Tanaka A, Hirabayashi K, Sadahiro S, et al. Inflammatory myofibroblastic tumor of the ascending colon in adults manifested by positive fecal occult blood test. Gastrointest Endosc 2010;71:214-216.

15. Salameh M, Sultan I, Barbar M, et al. Inflammatory myofibroblastic tumor causing unexplained anemia in a toddler: a case report. J Med Case Rep 2011;5:69.

16. Satahoo SS, Brathwaite C, Davis JS, et al. Obstructing apple core lesion of the rectum: a case report of inflammatory pseudotumor masquerading as colorectal carcinoma. J Pediatr Surg 2013;48:677-680.

17. Walia R, Gjikopulli A, Williams H, et al. Polypoid mass in the ascending colon with intussusception: a rare presentation of an inflammatory myofibroblastic tumor. J Pediatr Gastroenterol Nutr 2014;58:e35.

18. Przkora R, Bolder U, Schwarz S, et al. Regression of nonresectable inflammatory myofibroblastic tumours after treatment with nonsteroidal anti-inflammatory drugs. Eur J Clin Invest 2004;34:320-321. 